\title{
Microbial Genomics, DNA, and Pollution: What's Next?
}

\author{
J. T. Trevors
}

Published online: 13 October 2009

(C) Springer Science + Business Media B.V. 2009

\section{Introduction}

A Google scholar search of "microbial DNA" produced 657,000 returns. The same search under PubMed returned 27,718 articles. An immense amount of research has been undertaken and published on the subject of microbial DNA, genes, genomes, and methods to research microorganisms using a multitude of diverse methods. The structure and function of DNA was discovered followed by unraveling the genetic code, the capability to restrict and clone DNA in vectors and introduce the DNA into similar and different organisms, sequencing of DNA, and the polymerase chain reaction (PCR) to amplify DNA. Microarrays have been used to research DNA from a gene expression perspective, as well as real-time PCR to study gene expression. An obvious question is - what will researchers do next with DNA that will be a major advance in microbiology and other disciplines? Or posed in another way-what would you as a researcher like to do with DNA that you presently cannot accomplish because of technological limitations? The answers are challenging as researchers build on existing technologies and at the same time

J. T. Trevors $(\bowtie)$

School of Environmental Sciences, University of Guelph, Guelph, Ontario, Canada N1G 2W1

e-mail: jtrevors@uoguelph.ca improve and invent new technologies for researching DNA.

Many new microorganisms will be isolated and identified and their respective genomes sequenced. New genes and metabolic pathways will be discovered, and gene expression research will reveal the effects of diverse environmental conditions on gene expression. Site-directed mutagenesis will reveal knowledge about the role of specific mutations in the function of translated proteins. However, technologies to conduct this research are currently in place. The pressing question is what's next in microbial DNA technology?

The use of DNA technologies applied to microbiology research and environmental pollution is central to science, as microbes are the organisms that degrade the immense amounts of natural and human-made pollutants in our biosphere. Without microbes to degrade wastes/sewage, make compost, and degrade pollutants in soil and water, life would not be possible, as we know it on Earth. The biosphere cannot exist without the microbes that are central to our biogeochemical cycles. The importance of DNA technologies applied to microorganisms, pollution, biogeochemical cycles, and plant and animal pathogens cannot be overstated. The future will bring forth new DNA technologies and other technologies/discoveries that will allow a better understanding of microorganisms and their complex, diverse metabolic pathways that degrade much of the pollution in our biosphere. 